\title{
Introduction to Special Issue on Contextual Information Retrieval Systems
}

\author{
Fabio Crestani and Ian Ruthven \\ University of Strathclyde \\ Glasgow \\ G1 IXH \\ United Kingdom
}

Context affects all aspects of Information Retrieval. A searcher's context affects how they interact with a retrieval system, what type of response they expect from a system and how they make decisions about the information objects they retrieve. The information context is described by how these searchable objects are created, selected and linked within an information resource and context of use impacts on how we evaluate retrieval systems. Our understanding of user and information context also influences how we design and construct retrieval systems themselves.

The ability to respond to context could allow retrieval systems to learn and predict what information a searcher needs, decide how and when this information should be presented and distinguish between different search goals and searcher preferences. How the designers of search systems should respond to context is an important issue for the development of retrieval systems and the appropriate use of context raises many research questions. For example, what aspects of a searcher's context can we recognise, how should context be utilised within a retrieval system to improve search performance, how can we exploit shared contexts and contexts over time, and how should we evaluate contextual IR systems?

This special issue addresses the role of context in the theory and practice of IR systems development and the papers selected for this special issue demonstrate how contextual information can be used in a variety of ways to improve retrieval effectiveness. In these papers we see how system designers have responded in particular to the challenges of two important contextual factors: using context expressed within a domain and using the context embedded within specific work scenarios. Domains of expertise and knowledge create a context which relates concepts to one another and systems which can make use of these conceptual relationships for retrieval purposes can move beyond simple statistical processing of information to systems that support semantic retrieval. Three of the papers selected for this issue investigate the use of explicit knowledge structures, such as ontologies, for describing the context of specific domain areas.

The context of work tasks - the real-world information activities that promote searching - is also useful. Context in this case can either be an understanding of what information might be required for a particular task, such as shopping or medical diagnosis, or context can be the information objects used and created within the tasks. This task-based context can be usefully mined to suggest new documents to different people engaged in similar tasks. All the papers selected for this issue consider the nature of work tasks and how contextual retrieval systems can support task-based retrieval.

In our first paper, Campbell et al. consider the context of domain work tasks, that is the context of a knowledge worker engaged in a series of information tasks within a 
specific domain, and argue "one way in which an Information Retrieval system can respond to a searcher's current context is to evaluate the way the information that is currently in use had been used in previous circumstances." Campbell et. al.'s approach acknowledges and exploits contextual links between documents within a domain - documents either being linked based on their common use within a task or documents being used as the source in creating a new document. Their conceptual understanding of how people use documents within task contexts leads to a system to automatically detect contextual relationships based on users' interactions with documents. An experimental evaluation demonstrates how difficult it is to exploit contextual information but also how contextual information could be useful in supporting information tasks.

Hernandez et. al. examine how best to support domain-specific tasks through the use of contextual knowledge encoded within ontologies. Specifically they utilize two types of ontology - one representing thematic, or content, knowledge and one representing task information. Their approach is presented in a case study of astronomy research in which their task model contains of relationships centred on research tasks, e.g. authors can collaborate on an article, people work for institutions, articles can be published in journals or conferences, etc. whereas the thematic ontology deals with astronomical knowledge itself. These two areas can be combined in search tasks, e.g. "finding researchers having as domain interest a specific binary star" combines knowledge about astronomy and research activities. Through a careful and detailed exposition, Hernandez et. al. show how ontologies can be created and deployed within a system to provide a novel and flexible information access approach that supports a variety of user tasks.

Jung also investigates ontologies for knowledge representation. Here, however, the interest is in unifying heterogeneous web spaces - local ontologies in distinct websites - for use in the context of defined search tasks. Jung proposes an agent-based approach to gather information, represented as local ontologies, to support a user's information search. To overcome the diversity of language and topical content encoded in such local ontologies, and to provide a unified knowledge source, he shows how these local ontologies can be mediated to provide a personalised knowledge source. An experimental evaluation within the context of online shopping demonstrates effectiveness of such an approach.

Liu and Chu also consider supporting user work tasks by proposing a domain specific query expansion approach for what they call scenario-based retrieval: retrieval of information to support a specific use scenario. Within the medical domain they show how semantic, scenario-based approaches to query expansion differ from standard statistical approaches and how good query expansions for one scenario, e.g. treating a disease, may differ from those for a different scenario, e.g. diagnosing the disease. For scenarios that are poorly defined within a set of documents, they further propose a knowledge acquisition process to supplement existing resource with additional knowledge.

In each of these contributions we see, not only quality research papers, but conceptual approaches to utilising the idea of context in practical applications. 
We would like to thank the editors of Journal of Information Retrieval for their support in producing this special issue and the reviewers who gave their time and expertise in reading the submitted articles: Leif Azzopardi, Mark Baillie, Gloria Bordogna, Gobinda Chowdhury, Sándor Dominich, David Elsweiler, Joemon Jose, Henning Müller, David Losada, Jesper Schneider, Nicola Stokes, Theodora Tsikrika, Anastasios Tombros, Ryen White, and Enrique Herrera Viedma.

\section{References}

Campbell, D. R., Culley, S., McMahon, C. A., \& Sellini, F. An Approach for the Capture of Context-Dependent Document Relationships Extracted from Bayesian Analysis of User's Interactions with Information.

Hernandez, N. J., Mothe, J., Chrisment, C. \& Egret, D. Modeling Context Through Domain Ontologies.

Jung, J. J. Ontological Framework Based on Contextual Mediation for Collaborative Information Retrieval

Liu, Z, \& Chu, W. W. Knowledge-Based Query Expansion to Support ScenarioSpecific Retrieval of Medical Free Text. 